\title{
S Figure 1
}

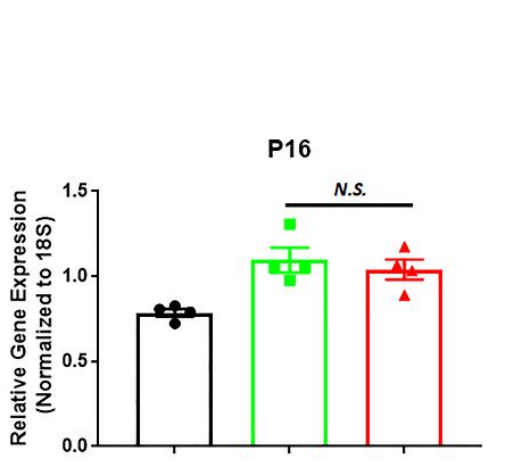

$$
\text { 口 Control }
$$

口 Condition Media_P7 HOBs

口Condition Media_P7 HOBs_BG
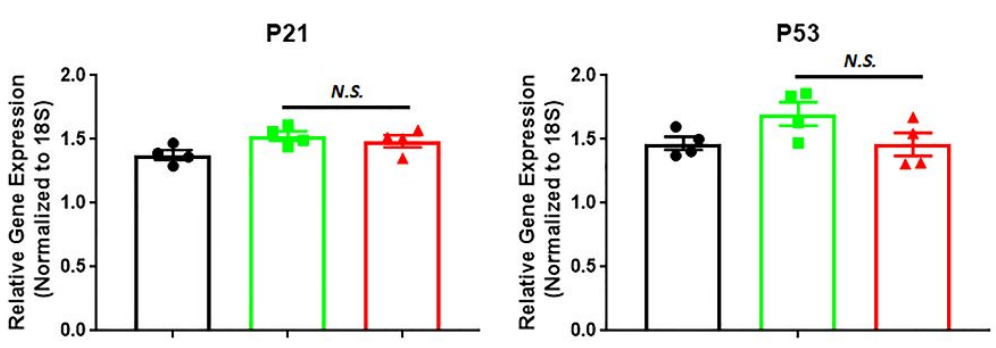

$\mathrm{IL}-1 \alpha$
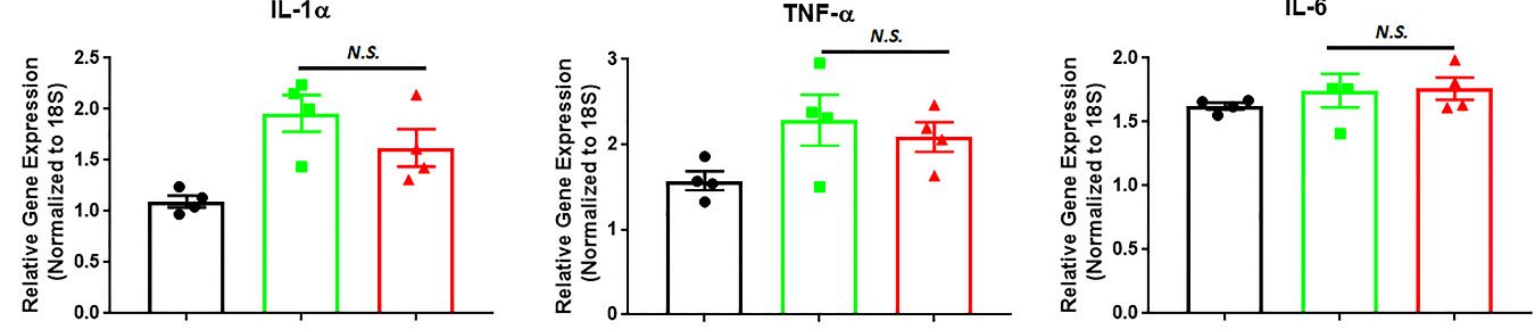

Supplementary Figure 1 The effects of soluble environment released from Baghdadite on

the modified paracrine effect of P7 HOB on P2 HOBs. The conditioned media from P7 HOBs was used to incubate Baghdadite and obtain the media extract (Conditioned media_P7 HOB_BD), which was then used to culture P2 HOBs. The conditioned media_P7 HOB_BD did not significantly affect the senescence-associated gene expression levels in P2 HOBs compared to those cultured in $\mathrm{P} 7 \mathrm{HOB}$ conditioned media. N.S.: Statistically significant difference was not observed, $P>0.05$ 


\section{HA/TCP}
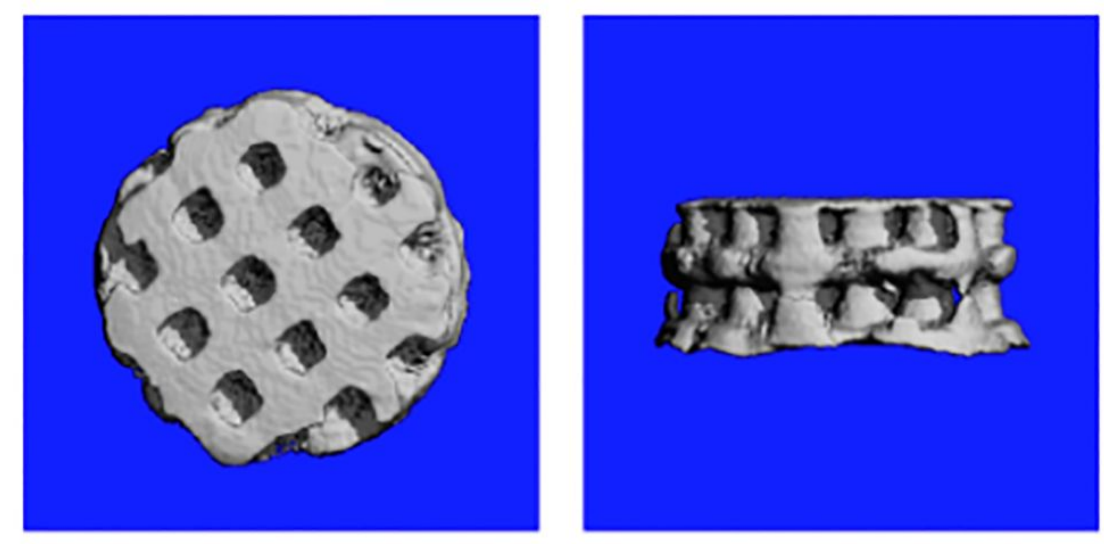

\section{Baghdadite}
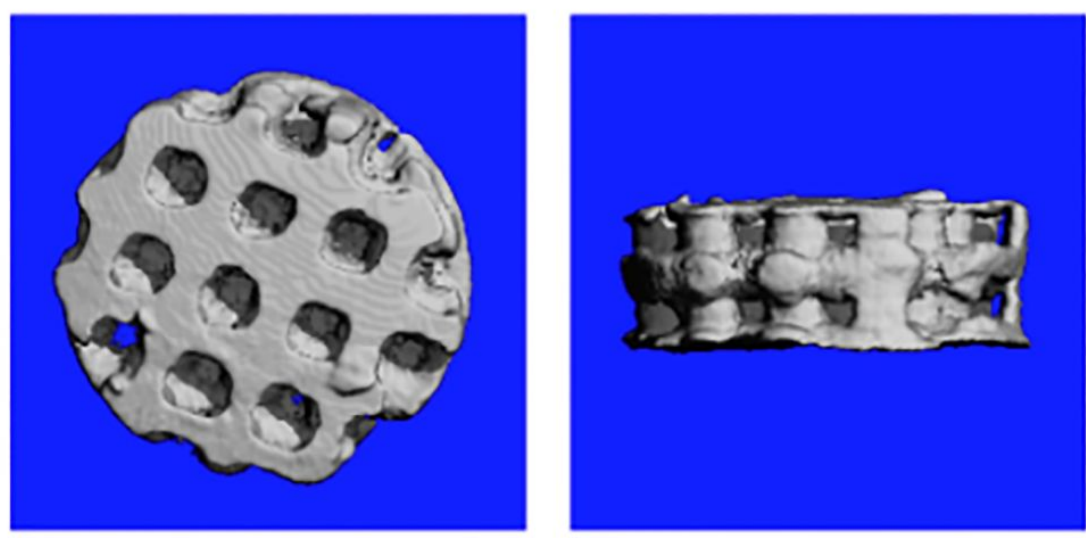

Supplementary Figure 2 Representative microCT images of 3D printed HA/TCP and

Baghdadite scaffolds. The scaffolds were fabricated through photolithography and characterized with the same pore size $(\sim 500 \mu \mathrm{m})$, porosity $(\sim 50 \%)$ and interior architecture. 